\title{
SYNTHESIS AND ANTIMICROBIAL STUDY OF NEW BENZIMIDAZOLE SCHIFF BASES BEARING P-TOLUENE SULFONAMIDE MOIETY
}

\author{
TURGAYTUNÇ * \\ Department of Chemistry Engineering and Process, Faculty of Engineering, University of Ahi Evran, Kirsehir, Turkey.
}

\begin{abstract}
Three new Schiff bases bearing benzimidazole and p-toluen sulfonamide moiety were synthesized. Their structures were characterized by elemental analysis, FTIR, NMR and LCMS methods. The antimicrobial properties of the compounds were also evaluated. All compounds exhibit higher antimicrobial activity against all bacteria, Schiff bases (4a-4c) shows more activity than the parent aldehyde (3). 4c displays higher antibacterial activity against $P$. aeruginosa compare to reference drug Sulfioxazole. The compounds were found more potent against Gram-negative than Gram positive bacteria. One more free phenyl group increase the activity in $4 \mathrm{c}$, but one more phenyl ring in $4 \mathrm{~b}$ does not.In vitro antifungal activity of the compounds was also tested on fungi C. albicans $C$. tropicalis and $C$. krusei. However, our compounds exhibit no antifungal activity against three fungi.
\end{abstract}

Keywords: Benzimidazole, Schiff bases, antimicrobial activity.

\section{INTRODUCTION}

Benzimidazoles are widely used group in the pharmaceutical industry because 5,6-dimethylbenzimidazole is a component of vitamin $\mathrm{B}_{12}$. Benzimidazole derivatives exhibits a wide range of biological properties including antimicrobial, antibacterial, antitumor, anticancer, anti-inflammatory and analgesic activities [1-14].

Sulfonamides interfere with the use of p-aminobenzoic acid (PABA) in the biosynthesis of tetrahydrofolic acid, which is an essential growth factor to the bacteria's metabolism [15]. Sulfonamides as artificial antifolic agents have been extensively used as antibiotic (Sulfa drugs). They are also have various pharmacological activities such as antifungal [16], antiviral [17], antitumor [18], anti-inflammatory [19]. Because of drug resistance, sulfonamides mainly used for the treatment of the urinary tract and methicillin resistant bacteria infections [20].

New antibiotics is a major global challenge in human-patogenic bacteria because of growing antibiotic resistance. In this context, some new sulfonamides having benzimidazole ring have been reported with their potential bactericidal and fungicidal agents against various bacterial and fungal resistant species [2123].

In order to give more benzimidazole-incorporated sulfonamide analogues, we synthesized new 2-methox-1- (4-methylbenzene-sulfonyl) benzimidazole (3) and its three Schiff base (4a-4c) derivatives, which is not reported in literature. Their antimicrobial and antifungal activities were also tested against three Gramnegative, three Gram-positive bacteria strains and three fungi.

\section{EXPERIMENTAL}

Melting points were measured by electrothermal IA9100 melting point apparatus in capillary tubes. The starting temperature of the approximate melting range was input via the keyboard and the melting point range was spotted visually. Elemental analyses for $\mathrm{C}, \mathrm{H}$ and $\mathrm{N}$ were carried out with an LECO CHNS-932 auto elemental analyzer. FT-IR spectra were recorded on a PerkinElmer spectrum and $\mathrm{KBr}$ pellets were used for solid samples. ${ }^{1} \mathrm{H}$ NMR and ${ }^{13} \mathrm{C}$ NMR spectra were determined on Bruker AV-400 using TMS (0.00 ppm) as internal standard and DMSO- $d 6$ as solvent.

\section{Synthesis of the Compounds}

\section{Chloromethyl-1H-benzoimidazole (1)}

1,2-Phenylenediamine $(0.5 \mathrm{~g}, 4.63 \mathrm{mmol})$ was dissolved in $5 \mathrm{M} \mathrm{HCl}$ solution, then chloroacetic acid $(0.51 \mathrm{~g}, 4.63 \mathrm{mmol})$ was added. The resulting mixture was refluxed for 15 hours at room temperature. $\mathrm{pH}$ of the solution was adjusted to 7.10 by adding concentrated ammonia, then yellow product was precipitated. Solid product was filtered, washed three times with distilled water, dried and crystallized in a mixture of ethyl alcohol and water. yield $80 \%$, e.n. $146-147^{\circ} \mathrm{C}$, [24].

\section{2-chloromethyl-1-(4-methylbenzene-sulfonyl) benzimidazole (2)}

2-Chloromethyl-1H-benzoimidazole (1 g, $5.98 \mathrm{mmol})$ was dissolved in acetonitrile and triethylamine $(0.97 \mathrm{~g}, 9.54 \mathrm{mmol})$ was added. The resulting mixture was poured into a salt-ice bath, than p-toluenesulfonyl chloride $(1.57 \mathrm{~g}$, $8.27 \mathrm{mmol}$ ) was added portion wise over 3 hours to the reaction medium. The mixture was stirred in a salt-ice bath for 4 hours. At the end of the reaction, the mixture was filtered and evaporated. The obtained solid was dissolved in ethyl alcohol and column chromatography was carried out in a 1: 5 ethylacetatepetroleum ether medium to obtain a substance with $80 \%$ yield; m.p $144-145{ }^{\circ} \mathrm{C}$ [25].

\section{2-methox-1-(4-methylbenzene-sulfonyl) benzimidazole (3)}

2-chloromethyl-1- (4-methylbenzene-sulfonyl) benzimidazole ( $0.5 \mathrm{~g}, 1.56$ $\mathrm{mmol})$ and 2-hydroxy benzaldehyde compound $(0.19 \mathrm{~g}, 1.56 \mathrm{mmol})$ dissolved in acetonitrile were added $\mathrm{K}_{2} \mathrm{CO}_{3}(2.15 \mathrm{~g}, 15.55 \mathrm{mmol})$ and $\mathrm{KI}(0.65 \mathrm{~g}, 3.92 \mathrm{mmol})$ were added. The resulting reaction mixture was stirred at room temperature for 5 days, then poured into saturated $\mathrm{NaHCO}_{3}$ solution and extracted 3 times with chloroform. The resulting organic phase was $\mathrm{Na}_{2} \mathrm{SO}_{4}$, then filtered and evaporated. The yellow 2-methox-1-(4-methylbenzene-sulfonyl) benzimidazole compound was obtained. Yield 80\%; m.p 141-142 ${ }^{\circ} \mathrm{C}$; LC-MS (m/z): 407.04 $[\mathrm{M}+\mathrm{H}]$ (calc. 406.46); Anal. calcd. for $\mathrm{C}_{22} \mathrm{H}_{18} \mathrm{~N}_{2} \mathrm{O}_{4} \mathrm{~S}$ : C, 65.01; $\mathrm{H}, 4.46 ; \mathrm{N}, 6.89$; Found: C, 65.89; H, 4.28; N, 6.26; FT-IR data (neat, v/ $\left./ \mathrm{cm}^{-1}\right): 1694.09(\mathrm{C}=\mathrm{O})$, 1598.71 (Ring C-C), 1479.42 (Ring C-C), 1452.34 (Ring $\mathrm{C}=\mathrm{N}$ ), 1191.08 ( $\mathrm{S}=\mathrm{O}$ ); ${ }^{1} \mathrm{H}-\mathrm{NMR}\left(\mathrm{DMSO}_{-} \mathrm{d}_{6}\right) \delta(\mathrm{ppm}): 2.4(3 \mathrm{H}), 3.3(2 \mathrm{H}), 7.21-8.13\left(\mathrm{~m}, 9 \mathrm{H}_{\mathrm{ar}}\right), 9.95(\mathrm{~s}$, $1 \mathrm{H}) ;{ }^{13} \mathrm{C}$ NMR (DMSO-d 6 ) $\delta$ (ppm): 21.78, 77.04, 113.05, 113.71, 120.99, $122.93,123.76,125.11,126.90,127.54,128.51,128.65,129.29,130.14,130.26$, $135.32,136.07,146.33,187.34$.

\section{2-((2-((1-tosyl-1-H-benzo[d]imidazol-2-yl)methoxy)benzylidene)amino) phenol (4a)}

2-methox-1-(4-methylbenzene-sulfonyl) benzimidazole compound was dissolved in ethyl alcohol $(0.25 \mathrm{~g}, 0.61 \mathrm{mmol})$ and 2-amino-phenol $(0.067 \mathrm{~g}, 0.61$ $\mathrm{mmol}$ ) was added. The reaction mixture was boiled under reflux for 24 hours then evaporated to give yellow 1H-benzimidazole, 1-[(4-methylphenyl) sulfonyl]-2-(phenoxy) methyl. Yield 55\%; m.p. 116-118 ${ }^{\circ} \mathrm{C}$; LC-MS (m/z): $497.99[\mathrm{M}+\mathrm{H}]$ (calc. 497.57); Anal. calcd. for $\mathrm{C}_{28} \mathrm{H}_{23} \mathrm{~N}_{3} \mathrm{O}_{4} \mathrm{~S}: \mathrm{C}, 67.59 ; \mathrm{H}, 4.66$; N, 8.45; Found: C, 67.08; H, 4.57; N, 8.29; FT-IR data (neat, v/ $\mathrm{cm}^{-1}$ ): 3057.27 $(\mathrm{O}-\mathrm{H}), 2974.95(\mathrm{C}-\mathrm{H}), 1597.70(\mathrm{C}=\mathrm{N}), 1488.67$ (Ring C-C), 1452.82 (Ring $\mathrm{C}=\mathrm{N}), 1161.06(\mathrm{~S}=\mathrm{O}) ;{ }^{1} \mathrm{NMR}\left(\mathrm{DMSO}_{\mathrm{d}}\right) \delta(\mathrm{ppm}): 2.35(3 \mathrm{H}), 3.40(2 \mathrm{H}), 7.28$ $7.59\left(\mathrm{~m}, 17 \mathrm{H}_{\mathrm{ar}}\right), 10.02(1 \mathrm{H}) ;{ }^{13} \mathrm{C}$ NMR (DMSO-d 6 ) $\delta(\mathrm{ppm}): 21.80,77.07,118.29$, $120.03,122.91,123.16,123.84,124.29,126.98,127.24,127.93,128.45,128.51$, $129.13,129.82,130.05,131.92,132.53,132.87,135.32,142.45,144.75,145.10$, $146.09,149.57,155.36,187.38$. 
2-((2-((1-tosyl-1-H-benzo[d]imidazol-2-yl)methoxy)napthylidene)amino) phenol (4b)

The compound was dissolved 2-methox-1-(4-methylbenzene-sulfonyl) benzimidazole $(0.25 \mathrm{~g}, 0.61 \mathrm{mmol})$ in ethyl alcohol and 3-amino-2-naphthol $(0.096 \mathrm{~g}, 0.61 \mathrm{mmol})$ was added to the solution. The reaction mixture was boiled under reflux for 24 hours then evaporated to give compound. Yield, 68\%; m.p. 118-120 ${ }^{\circ} \mathrm{C}$; LC-MS (m/z): $548.02[\mathrm{M}+\mathrm{H}]$ (calc. 547.63). Anal. calcd. for $\mathrm{C}_{32} \mathrm{H}_{25} \mathrm{~N}_{3} \mathrm{O}_{4} \mathrm{~S}$ : C, 70.18; H, 4.60; N, 7.67; Found: C, 71.04; H, 4.17; N, 7.46; FTIR data (neat, v/cm $\left.{ }^{-1}\right): 3053.82(\mathrm{O}-\mathrm{H}), 2973.67(\mathrm{C}-\mathrm{H}), 1625.87(\mathrm{C}=\mathrm{N}), 1486.40$ (Ring C-C), 1451.98 (Ring C=N), $1158.46(\mathrm{~S}=\mathrm{O}) ;{ }^{1} \mathrm{H}-\mathrm{NMR}$ (DMSO-d $\left.{ }_{6}\right) \delta(\mathrm{ppm})$ : $2.28(3 \mathrm{H}), 3.50(2 \mathrm{H}), 7.17-8.48\left(\mathrm{~m}, 19 \mathrm{H}_{\mathrm{ar}}\right), 9.43(1 \mathrm{H}) ;{ }^{13} \mathrm{C}$ NMR $\left(\mathrm{DMSO}^{-} \mathrm{d}_{6}\right) \delta$ (ppm): 21.77, 77.1, 119.93, 122.39, 123.25, 123.78, 125.45, 126.64, 127.53, $128.51,128.58,129.17,128.66,129.58,129.77,129.99,130.16,130.43,131.19$, $131.44,132.63,132.99,133.13,135.35,137.33,145.48,146.42,147.43,152.92$, 187.38.

1,2-diphenyl-2-((2-((1-tosyl-1-H-benzo[d]imidazol-2-yl)methoxy) napthylidene)amino)phenol (4c)

2-methox-1-(4-methylbenzene-sulfonyl) benzimidazole compound was dissolved in ethyl alcohol $(0.25 \mathrm{~g}, 0.61 \mathrm{mmol})$ and 2-Amino-1,2-diphenylethanol $(0.13 \mathrm{~g}, 0.61 \mathrm{mmol})$ was added. The reaction mixture was boiled under reflux for 24 hours then evaporated to give yellow compound. Yield, 75 , m.p. $148-150{ }^{\circ} \mathrm{C}$; LC-MS (m/z): 602.10 [M+ H] (calc. 601.72); Anal. calcd. for $\mathrm{C}_{36} \mathrm{H}_{31} \mathrm{~N}_{3} \mathrm{O}_{4} \mathrm{~S}: \mathrm{C}$, 71.86; H, 5.19; N, 6.98; Found: C, 71.94; H, 5.47; N, 6.73; FT-IR data (neat, $\left.\mathrm{v} / \mathrm{cm}^{-1}\right)$ : $3057.24(\mathrm{O}-\mathrm{H}), 1599.06(\mathrm{C}=\mathrm{N}), 1490.14$ (Ring C-C), 1454.96 (Ring $\mathrm{C}=\mathrm{N}), 1159.37(\mathrm{~S}=\mathrm{O}) ;{ }^{1} \mathrm{NMR}\left(\mathrm{DMSO}_{6} \mathrm{~d}_{6}\right) \delta(\mathrm{ppm}): 2.28(3 \mathrm{H}), 3.30(2 \mathrm{H}), 4.86$ $(1 \mathrm{H}), 5.38(2 \mathrm{H}), 6.86-7.99(\mathrm{~m}, 22 \mathrm{H}), 8.46(1 \mathrm{H}) ;{ }^{13} \mathrm{C} \mathrm{NMR}\left(\mathrm{DMSO}^{-} \mathrm{d}_{6}\right) \delta(\mathrm{ppm})$ : 21.30, 63.13, 76.68, 78.48, 116.94, 117.08, 117.12, 118.70, 118.88, 127.03, $127.22,127.40,127.66,127.79,127.87,128.06,128.10,128.17,128.41,128.54$, $128.72,128.78,129.03,129.59,129.80,131.46,131.70,131.84,132.45,132.57$, $132.78,139.21,139.49,140.17,160.92,165.95$.

\section{Antimicrobial activity Assay}

The antibacterial activity of the compounds was performed against American Type Culture Collection (ATCC) reference bacterial strain Salmonella typhimurium ATCC 14028, Staphylococcus aureus ATCC 25923 Enterococcus faecalis ATCC 29212, Escherichia coli ATCC 25922, Klebsiella pneumonia ATCC 27853, Pseudomonas aeruginosa ATCC 27853 cultures.

The compounds were dissolved in dimethylsulfoxide (10\%DMSO) to a final concentration of $8.0 \mathrm{mg} \mathrm{mL}^{-1}$ and sterilized by filtration with $0.45 \mathrm{~mm}$ millipore filters.

The minimum inhibitory concentration (MIC) values of the compounds were determined using modification of the micro well dilution assay method. $100 \mathrm{~mL}$ of the test compounds, initially prepared at $4000 \mathrm{mg} / \mathrm{mL}$ concentration, were added into the first wells. Then, $100 \mathrm{~mL}$ of the serial dilutions was transferred into nine consecutive wells. The contents of the wells were mixed and the micro plates were incubated at $37^{\circ} \mathrm{C}$ for $24 \mathrm{~h}$.

The compounds were tested against each microorganism twice. The values obtained are average of the two results. The MIC values were determined from visual examinations as the lowest concentration of the extracts in the wells with no bacterial growth. Sulfisoxazole were used as positive control.

Antimicrobial tests were also carried out by the disc diffusion method using $100 \mathrm{~mL}$ of suspension containing $10^{8} \mathrm{CFUmL}^{-1}$ bacteria which was spread on nutrient agar (NA) medium. The discs $(6 \mathrm{~mm}$ in diameter) impregnated with 30 $\mathrm{mL}$ of each compound $(240 \mathrm{mg} / \mathrm{disc})$ at the concentration of $8.0 \mathrm{mg} / \mathrm{mL}$ and placed on the inoculated agar. DMSO impregnated discs were used as negative control.

Sulfisoxazole $(300 \mathrm{mg} / \mathrm{disc})$ were used as positive control to determine the sensitivity of one strain/isolate in each microbial species tested.

The inoculated plates were incubated at $37{ }^{\circ} \mathrm{C}$ for $24 \mathrm{~h}$ for bacterial strains isolates. Antimicrobial activity in the disc diffusion assay was evaluated by measuring the zone of inhibition against the test organisms. Each assay in this experiment was repeated twice. Percentage of inhibition was calculated by comparing the distance of the sample to the distance of Sulfisoxazole as standard.
Table 1. Antimicrobial activity results of the compounds.

\begin{tabular}{|l|c|c|c|c|c|}
\hline Bacteria & \multicolumn{5}{|c|}{ MIC $(\boldsymbol{\mu g} / \mathbf{m L})$} \\
\hline & $\mathbf{3}$ & $\mathbf{4 a}$ & $\mathbf{4 b}$ & $\mathbf{4 c}$ & SD \\
\hline Gram-positive \\
\hline $\begin{array}{l}\text { S. aureus } \\
\text { ATCC 25923 }\end{array}$ & 250 & 62.5 & 62.5 & 31.25 & 23.4 \\
\hline $\begin{array}{l}\text { E. faecalis } \\
\text { ATCC 29212 }\end{array}$ & 250 & 62.5 & 62.5 & 31.25 & 93.75 \\
\hline $\begin{array}{l}\text { S. typhimurium } \\
\text { ATCC 14028 }\end{array}$ & 250 & 125 & 125 & 31.25 & 375 \\
\hline Gram-negative \\
$\begin{array}{l}\text { E. coli } \\
\text { ATCC 25922 }\end{array}$ & 125 & 31.25 & 31.25 & 31.25 & 23.4 \\
\hline $\begin{array}{l}\text { K. pneumonia } \\
\text { ATCC 27853 }\end{array}$ & 125 & 31.25 & 31.25 & 31.25 & 23.4 \\
\hline $\begin{array}{l}\text { P. aeruginosa } \\
\text { ATCC 27853 }\end{array}$ & 125 & 62.5 & 62.5 & 15.125 & 375 \\
\hline
\end{tabular}

SD: Sulfioxazole.

\section{RESULT AND DISCUSSION}

\section{Chemistry}

The new benzimidazole-incorporated sulfonamide derivatives were prepared from comercial 1,2-phenylenediamine and chloroacetic acid. Their synthetic routes were outlined in Scheme 1.

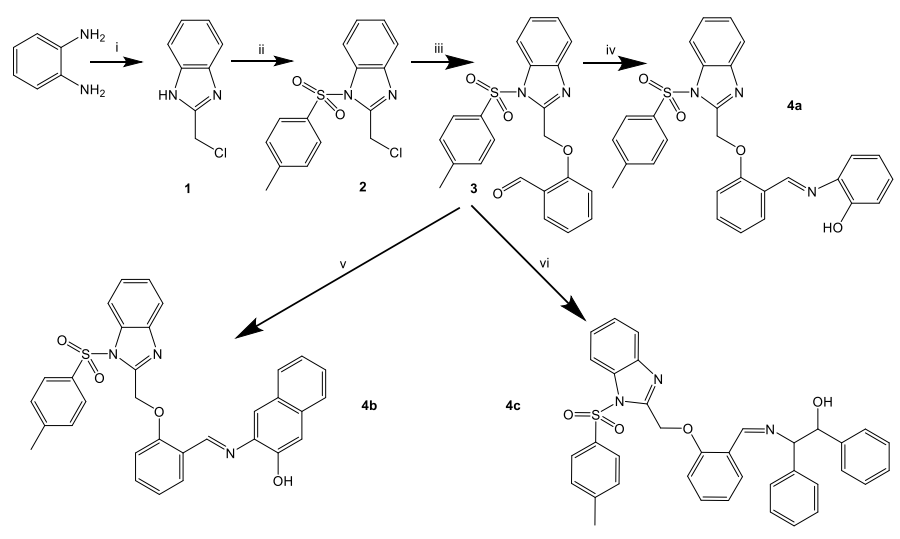

Scheme 1. Synthesis of benzimidazole-sulfonamide derivatives (3, 4a-4c).

Reagents and conditions: (i) chloroacetic acid, $25^{\circ} \mathrm{C}, \mathrm{pH} 7.1$ (ii) $\mathrm{p}$ toluenesulfonyl chloride, triethylamine, acetonitrile, $0{ }^{\circ} \mathrm{C}$, (iii) and 2-hydroxy benzaldehyde, $\mathrm{K}_{2} \mathrm{CO}_{3}, \mathrm{KI}$, acetonitrile, (iv) 2-amino-phenol, ethanol, (v) 3 amino-2-naphthol, ethanol, (vi) 2-Amino-1,2-diphenylethanol, ethanol.

\section{Evaluation of antimicrobial activity}

In vitro antibacterial activity of the compounds was screened on bacterial strains, Gram-positive $S$ aureus, E. faecalis, S. typhyimurium and Gram-negative E.coli, K. pneumonia,P.aeruginosa with microdilution method Antibacterial activity data (MIC) were given in Table 1 and 2. The order of antimicrobial activity is $3<4 a=4 b<4 c$. All compounds exhibit higher bacteriostatic activity against all bacteria, Schiff bases (4a-4c) shows more antimicrobial activity against all bacterial strains than the parent aldehyde (3). 4c displays higher antibacterial activity against $P$. aeruginosa compare to reference drug Sulfioxazole. The compounds were found more potent against Gram-negative than Gram positive bacteria. One more free phenyl group increase the activity in $\mathbf{4 c}$, but one more phenyl ring in $\mathbf{4 b}$ does not.

In vitro antifungal activity of the compounds was also tested on fungi $C$. albicans $C$. tropicalis and C. krusei. However, our compounds exhibit no antifungal activity against three fungi. 
Table 2. Disc diffusion antimicrobial activity results of the compounds.

\begin{tabular}{|c|c|c|c|c|c|}
\hline Bacteria & \multicolumn{5}{|c|}{ Zone diameter (mm 240 $\mathbf{\mu g}$ /disc) } \\
\hline \multicolumn{7}{|c|}{$\mathbf{3}$} & $\mathbf{4 a}$ & $\mathbf{4 b}$ & $\mathbf{4 c}$ & SD \\
\hline Gram-positive & \multicolumn{5}{|c|}{} \\
\hline $\begin{array}{c}\text { S. aureus } \\
\text { ATCC 25923 }\end{array}$ & 6 & 9 & 9 & 12 & 24 \\
\hline $\begin{array}{c}\text { E. faecalis } \\
\text { ATCC 29212 }\end{array}$ & 6 & 9 & 9 & 12 & 17 \\
\hline $\begin{array}{c}\text { S. typhimurium } \\
\text { ATCC 14028 }\end{array}$ & 6 & 10 & 10 & 12 & 10 \\
\hline Gram-negative & & & & & \\
\hline $\begin{array}{c}\text { E. coli } \\
\text { ATCC 25922 }\end{array}$ & 7 & 14 & 12 & 12 & 20 \\
\hline $\begin{array}{c}\text { K. pneumonia } \\
\text { ATCC 27853 }\end{array}$ & 7 & 14 & 12 & 12 & 27 \\
\hline $\begin{array}{c}\text { P. aeruginosa } \\
\text { ATCC 27853 }\end{array}$ & 7 & 9 & 9 & 16 & 8 \\
\hline
\end{tabular}

SD: Sulfioxazole.

\section{CONCLUSION}

In this study new four benzimidazole derivatives compounds were synthesis. The characterization of synthesis compounds was done by spectroscopic methods. The antimicrobial activities of compounds were determined and the results were compared to the Sulfioxazole compound. Among the synthesized compounds, $4 \mathrm{c}$ showed the strongest effect.

\section{REFERENCES}

1. Z.A. Alagoz, S. Y1ldız, E, Buyukbingol, Chemotherapy, 53, 110, (2007).

2. S.O. Podunavac-Kuzmanovic, D.M. Cvetkovic, J. Serb. Chem. Soc. 75, 459, (2007).

3. R.S. Keri, C.K. Rajappa, S.A. Patil, B.M. Nagaraja, Pharmacol. Rep, 68, 1254 (2016).

4. S.T. Asundaria, K.C. Patel, Pharm. Chem. J. 45, 725, (2012).

5. B. Soni, M.S. Ranawat, R. Sharma, A. Bhandari, S. Sharma, Eur. J. Med. Chem. 45, 2938, (2010).

6. A. Gellis, H. Kovacic, N. Boufatah, P. Vanelle, Eur. J. Med. Chem. 43, 1858, (2008).

7. H. M. Refaat, Eur. J. Med. Chem. 45, 2949, (2010).

8. K. Starcevic, M. Kralj, K. Ester, I. Sabol, M. Grce, K. Pavelic, G. KarminskiZamola, Bioorg. Med. Chem. 15, 4419, (2007).

9. S. Saeed, N. Rashid, P. G. Jones, M. Ali, R. Hussain, Eur. J. Med. Chem. 45, 1323, (2010).

10. D. Havrylyuk, L. Mosula, B. Zimenkovsky, O. Vasylenko, A. Gzella, R. Lesyk, Eur. J. Med. Chem. 45, 5012 (2010).

11. R. Caputo, M.L. Calabro, N. Micale, A.D. Schimmer, M. Ali, M. Zappala, S. Grasso, Med. Chem. Res. 21, 2644 (2012).

12. D. Evans, T.A. Hicks, W.R.N. Williamson, W. Dawson, S.C.R. Meacock, E.A. Kitchen, Eur. J. med. Chem. 31, 635 (1996).

13. M. Gaba, D. Singh, S. Singh, V. Sharma, P. Gaba, Eur. J. med. Chem. 45, 2245 (2010).

14. S. Bhattacharya, P. Chaudhuri, Current Medicinal Chemistry, 15, 1762 (2008).

15. M.E. Wolff, (Ed.), Burger's Medicinal Chemistry and Drug Discovery, 4rd ed.; John Wiley \& Sons: Laguna Beach, (1996).

16. J. Lal, S.K. Gupta, D. Thavaselvam, D.D. Agarwal, Eur. J. Med. Chem. 64, 579 (2013).

17. R. Gawin, E. De Clercq, L. Naesens, M. Koszytkowska-Stawinska, Bioorg. Med. Chem. 16, 8379 (2008).

18. V. Akurathi, L. Dubois, S. Celen, N.G. Lieuwes, S.K. Chitneni, B.J. Cleynhens, A. Innocenti, C.T. Supuran, A.M. Verbruggen, P. Lambin, G.M. Bormans, Eur. J. Med. Chem. 71, 374 (2014).

19. N. Chandna, S. Kumar, P. Kaushik, D. Kaushik, S.K. Roy, G.K. Gupta, S.M. Jachak, J.K. Kapoor, P.K. Sharma, Bioorg. Med. Chem. 21, 4581 (2013).

20. W.O. Foye, T.L. Lemke, D.A. Williams, Principles of Medicinal Chemistry, 4rd ed., Williams \& Williams, (1995).

21. X.J. Fang, P. Jeyakkumary, S.R. Avula, Q. Zhou, C.H. Zhou, Bioorg. Med. Chem. Lett. 26, 2584 (2016).

22. H.Z. Zhang, S.F. Cui, S. Nagarajan, S. Rasheed, G.X. Cai, C.H. Zhou, Tetrahedron Lett. 55, 4105 (2014).
23. J. Ponmani, H.B. Liu, L. Gopala, Y. Cheng, X.M. Peng, R.X. Geng, C.H. Zhou, Bioorg. Med. Chem. Lett. 27, 1737 (2017).

24. N. Siddiqui, Md.S. Alam, R. Ali, M. Shahar Yar, O. Alam. Med. Chem. Res., 25,1390 (2016).

25. K.B. Abdireimov, N.S. Mukhamedov, R. Ya Okmanov, M.Zh. Aiymbetov, B. Tashkhodzhaev, Kh.M. Shakhidoyatov, Chem. of Heterocyclic Compounds, 49, 760 (2013). 